\title{
Validation of Real-time Transthoracic 3D echocardiography in Children with Congenital Heart Disease
}

\author{
Authors: Annemien E. van den Bosch MD PhD \\ Derk-Jan Ten Harkel MD PhD, Jackie S. McGhie \\ Jolien W. Roos-Hesselink MD PhD, Ad J.J.C Bogers MD PhD, \\ Wim A. Helbing MD PhD and Folkert J. Meijboom MD PhD
}

Annemien E. van den Bosch, Jackie S. McGhie, Jolien W. Roos-Hesselink, Folkert J. Meijboom

Department of Cardiology, Erasmus MC University Hospital, Rotterdam, The Netherlands

\section{Ad J.J.C Bogers}

Department of Cardiothoracic Surgery, Thoraxcenter, Erasmus MC University Hospital, Rotterdam, The Netherlands

Derk-Jan Ten Harkel, Wim A. Helbing

Division of Paediatric Cardiology, Department Paediatrics, Erasmus MC University Hospital, Rotterdam, The Netherlands

Address for correspondence:

A.E. van den Bosch, MD

Department of Cardiology

Ba 300, Thoraxcenter

Erasmus MC University Hospital

Dr. Molewaterplein 40

3015 GD Rotterdam

The Netherlands

Email:

a.e.vandenbosch@erasmusmc.nl

ABSTRACT Aims Assessment of feasibility, accuracy and applicability in clinical practice of real-time three-dimensional echocardiography (RT-3DE) in children with congenital heart defects. Methods and results From September 2004 to June 2005, 100 consecutive children ( 57 infants, 43 children > I year of age), who were scheduled for corrective intracardiac surgery, were enrolled in this study. RT-3DE was performed with Philips Sonos 7500 echo-system and off-line analysis with TomTec Echoview( ${ }^{\circ}$ software. Quantitative and qualitative assessments of the region of interest were performed on the 3D reconstruction, comparing these results with the anatomic findings and measurements performed during intracardiac surgery. Acquisition of RT-3DE datasets was feasible in 92 of the 100 (92\%) patients and acquisition time was $6 \pm 3$ minutes. The overall quantitative analyses showed an excellent correlation $(r<0.90)$ between RT-3De and surgery. Also the qualitative analyses were accurate compared with surgical findings, in all patients in which RT-3DE was feasible. Conclusion This study shows that RT-3DE can be used in the clinical practice for the assessment of intracardiac anatomy in children with congenital heart disease. The information derived from the 3D reconstructions can be taken into consideration in the preoperative planning and management regarding interventional or surgical therapy.

\section{INTRODUCTION}

In a short time, real-time 3D echocardiography (RT-3DE) has developed into a clinically useful tool for the assessment of left ventricular (LV) volumes and function ${ }^{(1-5)}$. Recent experimental work has demonstrated that the current RT-3DE technique is also very promising for the assessment of intracardiac anatomy, not in the least because of its potential to present structural abnormalities in novel views $s^{(6-1)}$. The intracardiac anatomy is especially interesting in structural heart disease, of which congenital heart disease is the main contributor. Congenital heart disease often presents at neonatal or infant age, where accurate morphological and hemodynamic assessment is required as the basis of medical and/or surgical management. The question is whether the currently commercially available RT-3DE transducer is applicable in daily clinical practice in the paediatric population.

The RT-3DE transducer is not primarily developed for paediatric cardiology as expressed by low frequency $(2-4 \mathrm{MHz})$ of the transducer and large footprint. A low frequency is essential for use in the adult patients, but in conventional phased array transducers, it does not allow sufficient resolution of images in small paediatric patients. A larger footprint could be a problem when the transducer is placed in the narrow intercostal spaces of infants and young children.

We evaluated the feasibility, accuracy and possibility for clinical application of RT-3DE in children. One hundred children with a variety of congenital heart defects were imaged by RT-3DE, comparing the results to the anatomic findings at intracardiac surgery.

\section{METHODS}

Study population

From September 2004 to June 2005, 100 consecutive children (47\% male) with congenital heart disease, who were scheduled for corrective intracardiac surgery in our institute, were enrolled in this study. Of the 100 patients, 57 were infants (mean age $3.6 \pm 2.4$ months, body weight $5.1 \pm 3.0 \mathrm{~kg}$ ) and 43 were children (mean age $6.7 \pm 4.5$ years, body weight $22 \pm 12.9 \mathrm{~kg}$ ). Patients were excluded from the study if they were hemodynamically unstable, or acutely ill.The patient's characteristics may be viewed in Table I.

The study was explained to the patients and/or their parents who gave informed consent to be enrolled in the study. The institutional review board approved the study. 
TABLE I: Patient characteristics

\begin{tabular}{|c|c|c|c|c|}
\hline Diagnosis & $\mathrm{N}$ & Region of interest & Age & BW $(\mathrm{Kg})$ \\
\hline ASD \| & II & ASD & 4.5 y $(8 m-15 y)$ & $16.0(6.2-54)$ \\
\hline VSD & 23 & VSD & $4.8 m(1.5 m-15 y)$ & $5.2(2.4-31)$ \\
\hline ASD and VSD & 9 & ASD / VSD & $3.0 m(1.2 m-1 y)$ & $4.7(3-7.7)$ \\
\hline AVSD & 17 & AVSD & $4.8 m(1.2 m-16 y)$ & $5.1(3.1-49)$ \\
\hline ToF & 11 & VSD / RVOT & $3.6 m(1.5 m-15 y)$ & $5.2(2.4-31)$ \\
\hline Fontan & 7 & LVOT & $3 y(2 w k-9 y)$ & $14(2.6-30)$ \\
\hline AoS (subv/val) & 6 & LVOT & $7 y(8 m-12 y)$ & $24.0(9-34)$ \\
\hline VSD with AoS & 3 & VSD / LVOT & $3.5 y(3 y-16 y)$ & $15(12-60)$ \\
\hline VSD with PS & 3 & VSD / RVOT & $10 m(3 m-9 y)$ & $5.1(4.6-32)$ \\
\hline TGA & 3 & LVOT / ASD & $2 w k(1.5 w k-3 w k)$ & $3.5(3.5-4.4)$ \\
\hline PS & 4 & RVOT & $12 y(6 m-14 y)$ & $37.5(2.8-53)$ \\
\hline MV pathology & 2 & Inflow MV & $10 \mathrm{~m}(2.4 \mathrm{~m}, 1.5 \mathrm{y})$ & $5.7(3.5,7.8)$ \\
\hline Ebstein`s & 1 & Inflow TV & $7 y$ & 24 \\
\hline
\end{tabular}

Age and Body weight (BW) are expressed as median and range, $y=$ years; $m=$ months. ASD $\|=$ secundum atrial septal defect; VSD = ventricular septal defect; $A$ VSD = atrioventricular septal defect; $T$ ToF $=$ tetralogy of Fallot; $A 0 S=$ valvular of subvalvular aortic stenosis; TGA $=$ transposition of the great arteries; $P S=$ pulmonary stenosis; $M V=$ mitral valve; $L V O T=$ left ventricular outflow tract; RVOT = right ventricular outflow tract; $T V=$ tricuspid valve

\section{Three-dimensional echocardiographic data acquisition}

The real-time transthoracic 3D images were acquired with the Philips Sonos 7500 echo system (Philips Medical Systems, Andover, MA, USA) equipped with a $3 D$ data acquisition software package. The full matrix array (X4) transducer has several modes of data acquisition: I) narrow angle acquisition which consists of $60^{\circ} \times 30^{\circ}$ pyramidal volumes, displayed in a volume rendered manner.This is an actual real-time mode without the need for respiratory gating; 2) the "zoom mode" which allows a magnified view of a subsection of the pyramidal volume; and 3) wide angle acquisition which is used to collect a full volume data set of the heart. In this acquisition mode, four wedges are obtained over 8 consecutive cardiac cycles with ECG gating. The full matrix array $(X 4)$ transducer has a frequency range of $2-4 \mathrm{MHz}$.

For each individual patient a region of interest was defined. The region of interest is a part of the intracardiac anatomy that was inspected by the surgeon as a part of the surgical procedure. To evaluate different cardiac malformations, a list was made of the region of interest for the most common congenital heart malformations: atrial septal defect (ASD), ventricular septal defect (VSD), atrioventricular septal defect (AVSD), left ventricular outflow tract obstruction (LVOTO), right ventricular outflow tract obstruction (RVOTO), mitral valve (MV) and tricuspid valve (TV) abnormalities. In some patients, more than one region of interest could be evaluated.

The 3D data acquisition in the infants and children under the age of 4 years took place immediately before surgery, with the patients under general anaesthesia. During the data acquisition, no breath hold or ventilation arrest was performed. The patients were on conventional mechanical ventilation (tidal volume $8 \mathrm{ml} / \mathrm{kg}$ body weight). Since the data were acquired during the time that the child was prepared for surgery, there was no prolongation of total anaesthesia time. In all patients over 4 years of age $(n=24)$ a 3D data acquisition was performed without sedation, two weeks before surgery, during an outpatient clinic consultation.

\section{Three-dimensional echocardiographic data analysis}

The RT-3DE data were stored on a CD-ROM and transferred to a stand-alone TomTec workstation (TomTec, Munich, Germany) for offline data analysis with TomTec Echoview 5.2 ${ }^{\circledR}$ software. Threedimensional reconstructions, focused on the region of interest, were analyzed and interpreted off-line by two independent observers (AvdB and JM) unaware of the 2D echocardiographic findings. Acquisition time was defined as the total time for preparation - optimalisation of setting and transducer position - and the acquisitions of all the 3D data sets. The mean reconstruction time was defined as the time for screening all the 3D data sets for image quality and whether of not the entire region of interest was present in the data set, and making the necessary cut-planes in the best 3D data set for off-line analyses.

Qualitative analysis: The intracardiac structures were analysed by making cut planes through the 3D data set in order to get an optimal visualisation of the region of interest. These cut planes can be made at any desirable angle. The images were rotated in space to visualise the intracardiac structures from different sides.

For analysis of the atrial and/or ventricular septal defect, a cut plane was placed in the 3D data set through the LV and RV from apex to base, parallel to the ventricular septum, creating an "en face" view of the septal surface. For analysis of the AV valves (AVSD, MV and/or TV pathology), two opposite views were reconstructed. The "unroofed" view from the left atrium orientated toward the atrioventricular junction creating a "surgical view" of the AV valve. Similar reconstruction, but orientated from the LV apex toward the AV junction, allowed the assessment of the $A V$ valve from below. The number, size and chordal attachment of the AV valve leaflets, especially to the crest of the interventricular septum were identified. In cases of LVOT or RVOT obstruction, the outflow tract was assessed from long axis and short axis cut planes at different angles and distances, from below and above the obstruction. The morphology of the sub- or valvular obstruction, its extension and relation to the aortic or pulmonary valve were assessed. The aortic and pulmonary valves were evaluated for the number of cusps and the fusion of the commissures.

Quantitative analysis: Quantitative analysis was performed for measurements of distance and diameters. Septal defects - ASD, VSD and AVSD - were assessed for the maximal diameter and compared with surgical measurements. Also the shape and number of defects were determined. In LVOT, RVOT,MV andTV abnormalities, the maximal annulus diameter was measured on 3D image. 
Surgical procedure and assessment of morphology

The surgeon was blinded for the RT-3DE study to avoid bias in the surgical report. Measurements and descriptions were obtained, in the flaccid heart after cold cardioplegia (St. Thomas solution). Using the surgical description as the gold standard, the accuracy of each type of echo assessment was determined.

\section{STATISTICAL ANALYSIS}

All values are expressed as means \pm standard deviation (SD) when data were normally distributed. Data were tested for normality with the Kolmogorow-Smirnov test. The median and range are given when data were distributed asymmetrically. The relations between diameters derived from RT-3DE and surgical procedure, and interobserver variability were analysed by linear regression and Bland-Altman analysis ${ }^{(12)}$. Limit of agreement is expressed as 2 SDs of the difference between RT-3DE and surgery. To determine whether the difference in the values between RT-3DE and surgical measurements was statistically significant, a paired t-test was performed. A p-value less than .05 was considered significant.

\section{RESULTS}

Different regions of interest were selected according to the specific congenital defects. Acquisition of RT-3DE datasets was feasible in 92 of the 100 (92\%) patients. There were 4 patients with poor acoustic windows (age I - 3 years) and 4 patients (age 6,7,7 and 10 months) with persistent respiratory artefacts that precluded adequate $3 \mathrm{D}$ rendering. The time of 3D data acquisition was $6 \pm 3$ minutes. In all infants and young children the subcostal view was found to be the best approach giving an unobstructed acoustic window for creating an adequate 3D

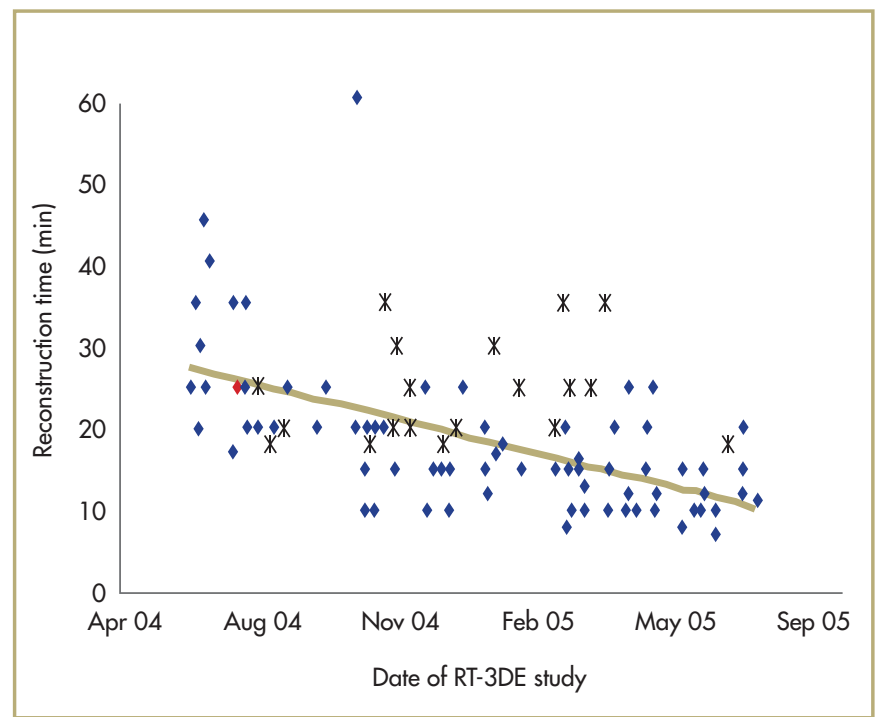

FIGURE 1 represents our learning curve for 3D reconstruction during the study. - = patients with simple congenital heart disease (including atrial septal defect, ventricular septal defect and atrioventricular defect);

X = patients with complex congenital heart disease (including all other congenital heart malformations). data set for any of the defined regions of interest. This window was sufficient to enclose the entire heart including the region of interest in one data set. For the children above the age of 4 years, the transducer position was adjusted in order to have the ultrasound beam perpendicular to the region of interest, resulting in some unorthodox transducer positions. The overall reconstruction time is shown in Figure I.

\section{REGION OF INTEREST}

\section{Atrial septal defects}

RT-3DE acquisition was feasible in 20 of the 21 (95\%) patients. Realtime 3D echocardiographic evaluation of 17 secundum ASDs and 3 sinus venosus defects provided unique enface views of the defect from the right atrial (RA) as well as the left atrial $(L A)$ sides and its spatial orientation (Figure 2). The mean reconstruction time was $15 \pm 4$ minutes, but became significantly shorter during the course of the study as experience improved. Maximum ASD diameter was $17 \pm 11 \mathrm{~mm}$ by RT-3DE and $18 \pm 11 \mathrm{~mm}$ by surgery $(P=0.1)$. Minimum ASD diameter was $\mathrm{I} I \pm 5 \mathrm{~mm}$ by RT-3DE and $\mathrm{I} \pm \pm 6 \mathrm{~mm}$ by surgery $(P=0.3)$. The correlation between RT-3DE and surgery for the maximum ASD diameter was $r=0.97(y=0.93 x+0.29 ;$ SEE $=2.6 \mathrm{~mm})$ with a mean difference of $1.0 \pm 5.0 \mathrm{~mm}$.

FIGURE 2: Real-time 3D echocardiographic display of sinus venosus defect of the superior vena cava type.

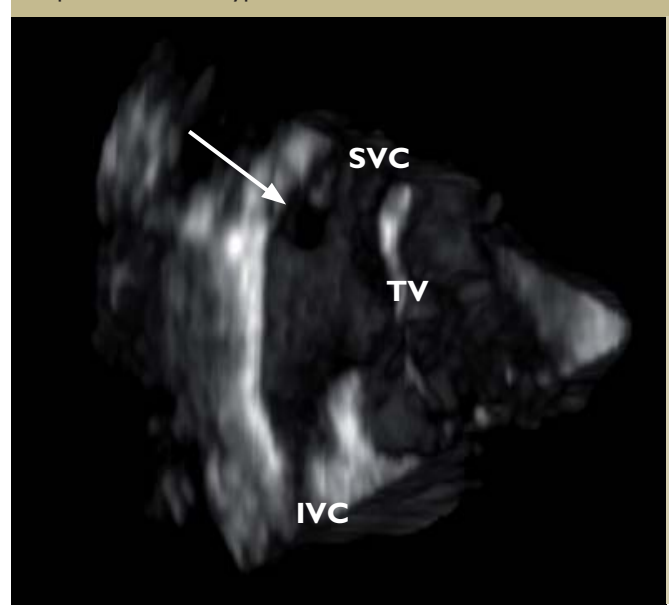

A: Viewed from right atrial surface in a patient of 5 years old.

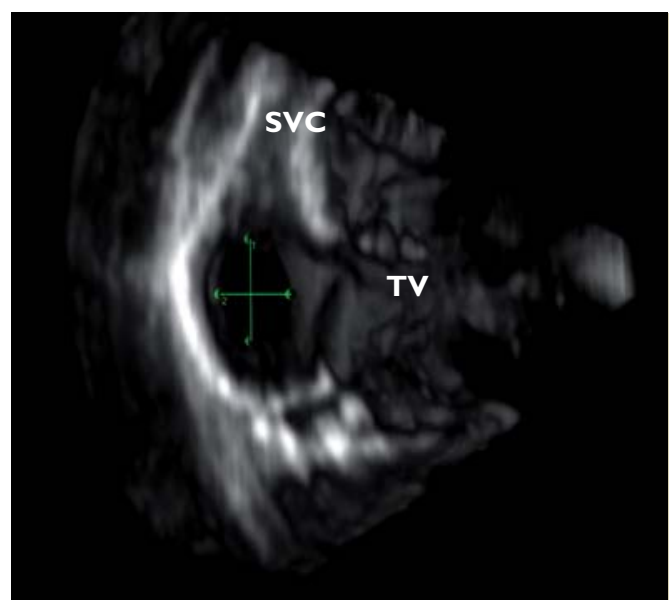

B: Shows the diameter measurement of a secundum atrial septal defect. 


\section{Ventricular septal defects}

RT-3DE acquisition was feasible in 40 of the 45 (89\%) patients (23 patients with isolated VSD, 9 patients with ASD and VSD, and 13 patients with VSD and PS/AoS orToF). In all patients, the free wall of the $\mathrm{RV}$ was digitally resected from the $3 \mathrm{D}$ data set to visualise the right side of the ventricular septum (surgical view). The mean reconstruction time was $17 \pm 10$ minutes. MaximumVSD diameter was $11.5 \pm 3.7 \mathrm{~mm}$ and $11.9 \pm 4.6 \mathrm{~mm}$, for respectively RT-3DE and surgery $(P=0.3)$. MinimumVSD diameter was $6.9 \pm 2.9 \mathrm{~mm}$ and $9.6 \pm 4.3$, for respectively RT-3DE and surgery $(P<0.00 I)$. The correlation between RT-3DE and surgery for the maximum VSD diameter was $r=0.94(y=0.84 x+$ 1.79; $\mathrm{SEE}=1.5 \mathrm{~mm}$ ) with a mean difference of $0.1 \pm 2.8 \mathrm{~mm}$. The measurements performed by the two observers did not differ significantly. The interobserver variability showed a good correlation for the VSD measurements $(r=0.93 ; y=0.97 x+0.31)$ with a mean difference of $0.4 \pm 1.5 \mathrm{~mm}$.

\section{Atrioventricular defects}

RT-3DE acquisition was feasible in all 17 patients ( 10 patients with a common AV orifice (complete AVSD), 7 patients with divided AV orifices (partial AVSD)). In all 17 patients, the AV valve leaflets were assessed correctly compared with the surgical findings. In 16 patients, $5 \mathrm{AV}$ valve leaflets were identified and one patient had $4 \mathrm{AV}$ valve leaflets (Figure 3). The superior bridging leaflet was assessed on the RT-3DE image according to the Rastelli classification: 7 patients with Rastelli type A and 3 patients with Rastelli type C. The RT-3DE observations of the superior bridging leaflet were accurate compared with the findings during surgery.

\section{Left ventricular outflow tract and/or aortic valve pathology}

In 12 of the 13 (92\%) patients a reconstruction of this region of interest was performed. In 5 patients, a subvalvular stenosis was assessed by 3D reconstruction looking from the LV up to the LVOT. The diameter of the LVOT was reduced for 25 - 50\% in 4 patients by a fibrous crescent or ring; in one patient the obstruction was almost $90 \%$. The distance from the base of the obstruction to the aortic valve was $9 \pm 1.3 \mathrm{~mm}$ These findings were confirmed by surgery. In 3 patients, severe aortic regurgitation was present and one patient had severe aortic stenosis. In the $3 \mathrm{D}$ reconstruction of aortic valve, the closure line was clearly visible, but often not the separate cusps. In the 3 patients with transposition of the great arteries, the parallel arrangements of the great arteries and spatial relations could simultaneously be visualised.The LVOT obstruction and arrangement of the great arteries were correctly assessed by RT-3DE compared with surgery.

Right ventricular outflow tract defect and/or pulmonary valve pathology

Three-dimensional reconstructions of the RVOT were performed in 10 patients with subvalvular obstruction. Infundibular hypertrophy was present in 9 patients and one patient had pulmonary atresia. The
FIGURE 3: Real-time 3D echocardiographic display of a partial (A) and complete AVSD (B) in respectively a patients of 3 years and I month old.

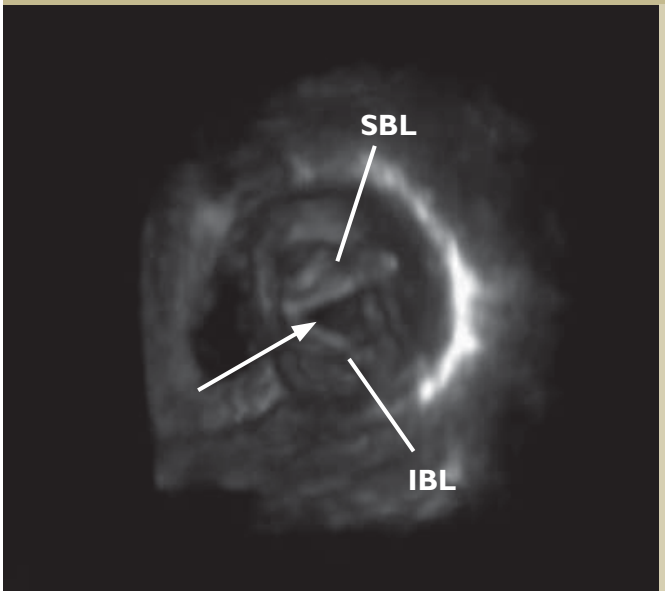

A: Shows the commissure (arrow) between the superior (SBL) and inferior bridging leaflets (IBL) in a partial AVSD, viewed from the LV apex.

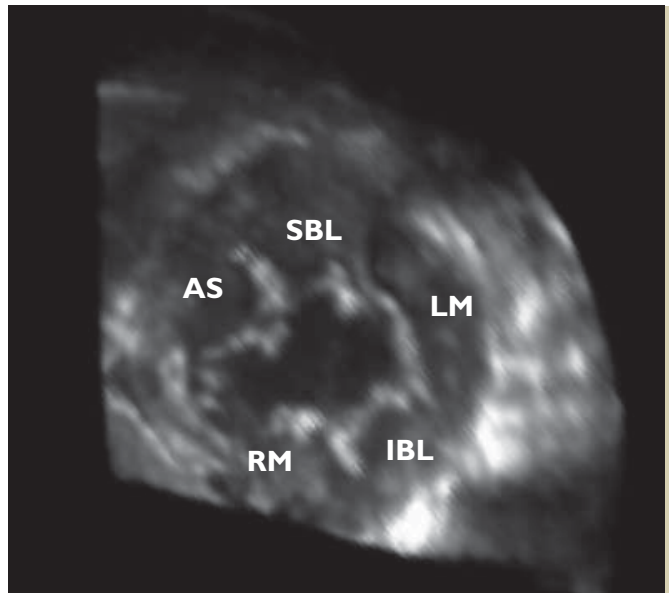

B: Shows the opening of the common AV valve in a patient with a complete AVSD. The 5 AV valve leaflets are clearly identified.

$A S=$ antero-superior leaflet; $R M=$ right mural leaflet; $L M=$ left mural leaflet

smallest RVOT diameter was $6.4 \pm 1.1 \mathrm{~mm}$ and $5.6 \pm 1.5 \mathrm{~mm}$, for respectively $R T-3 D E$ and surgery $(P=0.2)$. The pulmonary valve was assessed in 17 of the 20 (85\%) patients. The closure line of the pulmonary valve was visible, but not the pulmonary valve cusps. The maximal pulmonary valve annulus was $8 \pm 5 \mathrm{~mm}$ and $8 \pm 5 \mathrm{~mm}$, for respectively $R T-3 D E$ and surgery $(P=0.2)$.

\section{Mitral or tricuspid valve pathology}

Tricuspid or mitral valve was evaluated in 7 patients (3 patients with hypoplastic LV, two patients with mitral valve stenosis and regurgitation, one patient with Ebstein's anomaly and one patient with double outlet RV). Real-time 3D echocardiography provided "en face" view of all leaflets at any point in the cardiac cycle by single acquisition (Figure 4). The TV and MV annulus could be measured in all 7 patients.

\section{DISCUSSION}

This study demonstrates that the currently commercially available RT-3DE is feasible in infants and children, and accurately depicts the intracardiac morphology of congenital heart malformations. Acquisition time is short and reconstruction time is, after a brief learning curve, 


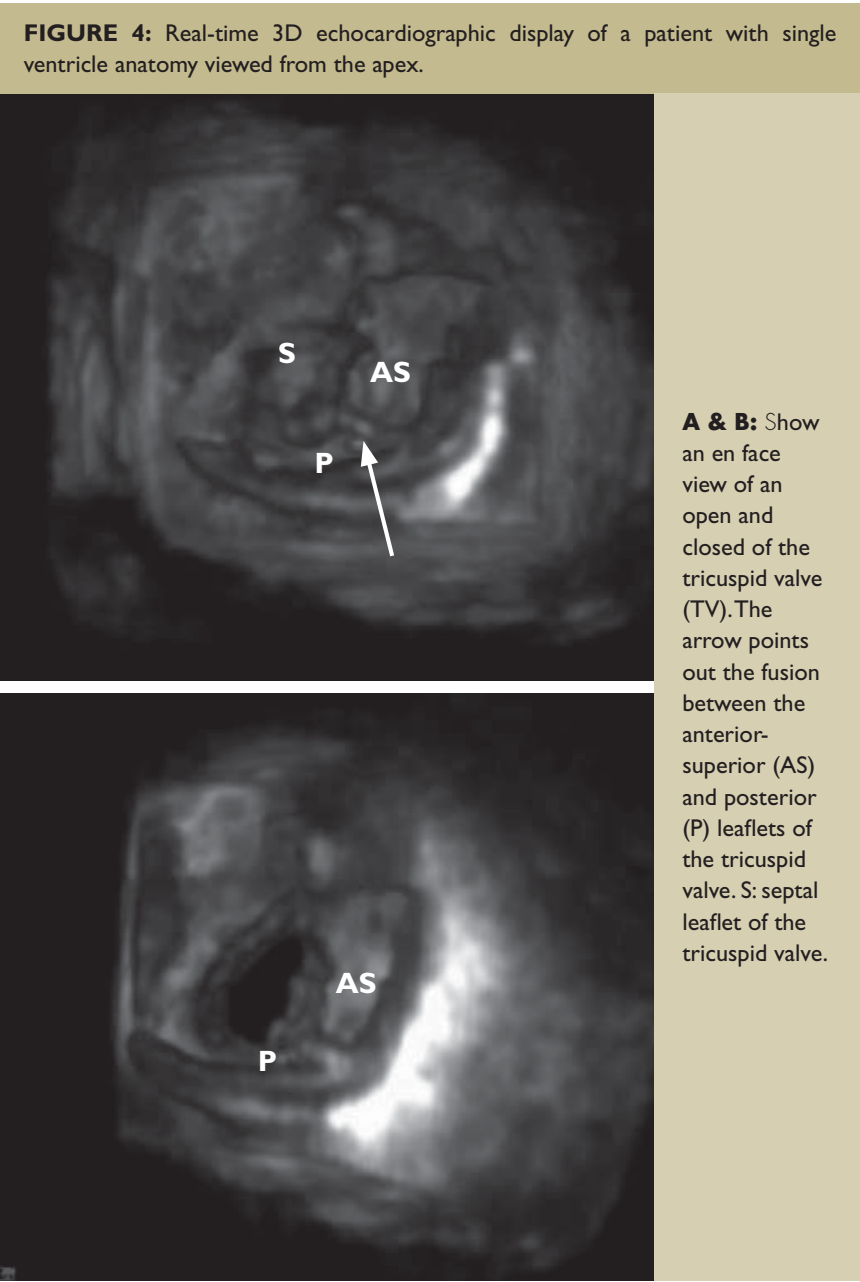

acceptable. This implicates that RT-3DE is no longer a research tool, but can be used in the daily clinical practice of paediatric cardiology.

At this moment, 2D echocardiography is the most commonly used non-invasive diagnostic method for analysis of congenital heart defects. However, when information about the spatial relationship of the cardiac structures is necessary, 2D echocardiography has its obvious shortcomings, as it rarely displays the anatomy in views that are similar to the ones encountered during surgery. It can only provide (multiple) 2D imaging planes, which requires a difficult mental conceptualization process of the viewer to create a 3D image of the intracardiac anatomy. In this study, we were able to diagnose the cardiac malformation in 92\% of patients studied with the RT-3DE technique, providing us with depth perception of cardiac structures; now really showing their relation with surrounding structures and generating "en face" views such as the surgical views.

For example, RT-3DE can delineate atrial- and ventricular septal defects for localization, size and relation to relevant structures during the cardiac cycle ${ }^{(8,13,14)}$. With the development of new techniques for septal defect closure, the need for accurate assessment of not only defect size but also defect morphology and its spatial relations increases ${ }^{(15,16)}$. This unique presentation of the intracardiac anatomy, validated in this study, is the main advantage of RT-3DE over the existing I-D and 2-D mode.

A great advantage of RT-3DE is the applicability in infants and young children. The inability of a proper breath hold and use of sedation in young children could limit the clinical application. However, sedation is often used when performing 2D echocardiograms in infants and children younger than 3 years to limit motion artifacts and to ensure a complete echocardiographic examination ${ }^{(17,18)}$. We expect that sedation is not necessary in calm lying neonates and young children, for example during feeding or sleep, because RT-3DE acquisition time is very short.

A learning curve was necessary before it was possible to obtain an adequate $3 \mathrm{D}$ data set. We assumed that one 3D data set acquired from one view, would comprise all data and that it would be possible to analyse the entire heart. But It is impossible to see all intracardiac structures with the same optimal image quality in one data set ${ }^{(19,20)}$. The reduced lateral resolution, far and near field problems of the matrix transducer hampers the image quality of structures in these parts of the 3D data set. To ensure good image quality, we adjusted our scanning technique in such a way that the ultrasound beam was always perpendicular to the region of interest and that this region of interest was in the centre of the 3D data set. For infants and young children, the best approach was the subcostal view; however, for children above 4 years of age standard transducer positions required adjustment, resulting in unorthodox scanning planes.

The highest frequency transducer currently available is $2-4 \mathrm{MHz}$, which in the conventional 2D transducer precludes good image resolution in small paediatric patients. Despite this low frequency, we were able to obtain adequate 3D data set and found that the resolution was much better than expected. Even small intracardiac structures such as chordae and papillary muscles could be visualized. An explanation for the better lateral resolution could be the large number of crystals in the matrix transducer. The crystals are parallel processed, allowing a significant increase in the ratio of transmitted to received ultrasound signals. The currently available matrix transducer has a relative large footprint that prohibits undistributed echo window in the small intercostal spaces of infants and young children. In our experience, the optimal acoustic window in neonates and small children is the subcostal view, making the drawback of large footprint less relevant.

We believe that with further refinement of the 3D matrix technology, such as the development of a high frequency transducer and smaller footprint, 3D echocardiography is likely to play a valuable role in the analysis of congenital heart disease and could aid in the choice in the preoperative planning by providing undisputed presentation of the defect's morphology and spatial orientation on top of the conventional 2D echocardiography. 


\section{LIMITATIONS OF REAL-TIME 3D ECHOCARDIOGRAPHY}

Although RT-3DE has great potential, it is still limited by less resolution when compared with 2D echocardiography.Very thin cardiac structures such as aortic and pulmonary leaflets are difficult to visualize, even with optimal gain settings, and dropouts in the 3D data set are common. An additional limitation of the RT-3DE includes the limited size of the pyramidal volume of the data set and irregular heart rate for full volume $3 \mathrm{D}$ acquisition that can prolong acquisition time and degrade the quality of the 3D image.

Three-dimensional data acquisition in the infants and children under the age of 4 years was performed under general anaesthesia. Therefore, the outcomes of this study cannot directly be extrapolated to the use of RT-3DE in clinical practice for the study of young children without sedation.

\section{CONCLUSION}

Real-time 3D echocardiography has great potential in the assessment of intracardiac anatomy, so important in congenital heart disease. This study shows that, despite the shortcoming of the currently commercially available RT-3DE equipment in terms of limited image resolution and large footprint of the transducer, RT-3DE can be used in the clinical practice for the assessment of intracardiac anatomy in infants and children with congenital heart disease. The information derived from the $3 \mathrm{D}$ reconstructions can be taken into consideration in the preoperative planning and management regarding interventional or surgical therapy.

\section{REFERENCES:}

I. Kuhl HP, Schreckenberg M, Rulands D, Katoh M, Schafer W, Schummers G, Bucker A Hanrath P, Franke A. High-resolution transthoracic real-time three-dimensional echocardiography: quantitation of cardiac volumes and function using semi-automatic border detection and comparison with cardiac magnetic resonance imaging. I Am Coll Cardiol. 2004:43:2083-90.

2. Jenkins C, Bricknell K, Hanekom L, Marwick TH. Reproducibility and accuracy of echocardiographic measurements of left ventricular parameters using real-time threedimensional echocardiography. J Am Coll Cardiol. 2004:44:878-86.

3. Fei HW,Wang XF, Xie MX, Zhuang L, Chen LX, Yang Y, Huang RQ.Wang J.Validation of real-time three-dimensional echocardiography for quantifying left and right ventricular volumes: an experimental study. Chin Med J (Engl). 2004;1 17:695-9.

4. Arai $K$, Hozumi T, Matsumura Y, Sugioka $K$, Takemoto $Y$, Yamagishi H, Yoshiyama M, Kasanuki H, Yoshikawa J. Accuracy of measurement of left ventricular volume and ejection fraction by new real-time three-dimensional echocardiography in patients with wall motion abnormalities secondary to myocardial infarction. Am 」 Cardiol. 2004;94:552-8

5. Hart JP, Cabreriza SE, Gallup CG, Hsu D, Spotnitzt HM.Validation of left ventricular enddiastolic volume from stroke volume and ejection fraction. Asaio J. 2002:48:654-7.

6. Van den Bosch AE, Van Dijk VF, McGhie JS, Bogers AJ, Roos-Hesselink JW, Simoons ML, Meijboom FJ. Real-time transthoracic three-dimensional echocardiography provides additional information of left-sided AV valve morphology after AVSD repair. Int J Cardiol. 2005.

7. Cheng TO, Xie MX,Wang XF,Wang Y, Lu Q. Real-time 3-dimensional echocardiography in assessing atrial and ventricular septal defects: an echocardiographic-surgical correlative study. Am Heart 1. 2004; 1 48:1091-5.

8. Marx GR,Sherwood MC,Fleishman C,Van Praagh R.Three-dimensional echocardiography of the atrial septum. Echocardiography. 200 I; 1 8:433-43.

9. Espinola-Zavaleta N,Vargas-Barron J, Keirns C, Rivera G, Romero-Cardenas A, Roldan 」, Attie F.Three-dimensional echocardiography in congenital malformations of the mitral valve. J Am Soc Echocardiogr. 2002; 15:468-72.

10. Salehian $\mathrm{O}$, Chan KL. Impact of three-dimensional echocardiography in valvular heart disease. Curr Opin Cardiol. 2005;20:122-6.

II. Schnabel R, Khaw AV,Von Bardeleben RS, Strasser C, Kramm T, Meyer J, Mohr-Kahaly S. Assessment of the tricuspid valve morphology by transthoracic real-time-3Dechocardiography. Echocardiography. 2005;22:15-23.

12. Bland JM, Altman DG. Statistical methods for assessing agreement between two methods of clinical measurement. Lancet. 1986; 1:307- I0.

13. Acar P, Roux D, Dulac Y, Rouge P, Aggoun Y. Transthoracic three-dimensional echocardiography prior to closure of atrial septal defects in children. Cardiol Young. 2003; | 3:58-63.

14. Dall'Agata A, Cromme-Dijkhuis AH, Meijboom FJ, McGhie JS, Bol-Raap G, Nosir YF Roelandt IR, Bogers AJ.Three-dimensional echocardiography enhances the assessment of ventricular septal defect. Am J Cardiol. 1999:83: I576-9, A8

15. Acar P, Abdel-Massih T, Douste-Blazy MY, Dulac Y, Bonhoeffer P, Sidi D. Assessment of muscular ventricular septal defect closure by transcatheter or surgical approach: a three-dimensional echocardiographic study. Eur J Echocardiogr. 2002;3: | 85-91.

16. Chessa M, Carminati M, Cao QL, Butera G, Giusti S, Bini RM, Hijazi ZM. Transcatheter closure of congenital and acquired muscular ventricular septal defects using the Amplatzer device. J Invasive Cardiol. 2002; | 4:322-7.

17. Ghaffar S, Haverland C, Ramaciotti C, Scott WA, Lemler MS. Sedation for pediatric echocardiography: evaluation of preprocedure fasting guidelines. J Am Soc Echocardiogr. 2002;15:980-3.

18. Napoli KL, Ingall CG, Martin GR. Safety and efficacy of chloral hydrate sedation in children undergoing echocardiography. J Pediatr. |996; |29:287-9|

19. Kasliwal RR, Chouhan NS, Sinha A, Gupta P, Tandon S, Trehan N. Real-time threedimensional transthoracic echocardiography. Indian Heart J. 2005;57: I 28-37.

20. Cotrim C, Cordeiro P, Zamorano J, Simoes O, Loureiro MJ, Vinhas H, Oliveira M, Carrageta M. Adolescent and adult congenital heart disease assessed by real-time three-dimensional echocardiography: an initial experience. Rev Port Cardiol. 2005;24:547-53. 